\title{
Stationary Distribution and Periodic Solution for the Stochastic Chemostat with Perturbations on Multiple Parameters
}

\author{
Zhen $\mathrm{Li}$ \\ College of Science, University of Shanghai for Science and Technology, Shanghai 200093, P. R. China \\ Email: 1145146074@qq.com
}

\begin{abstract}
We describe the periodic dilution rate and periodic mortality of a stochastic chemostat model with the response of the Monond function. We consider that both nutrient's input concentration and the death rate of microbe are simultaneously influenced by white noise. For this chemostat, we have found sufficient conditions which have a stationary distribution and stochastic nontrivial positive periodic solution.
\end{abstract}

Keywords: Stochastic chemostat model; Itô's formula; Nontrivial positive periodic solution

\section{Introduction}

The chemostat is a continuous culture device that manages to keep the flow rate of culture medium constant and the growth rate of microorganism is always lower than its growth rate [1]. Experimenters usually use the appropriate device to limit the concentration of nutrients in the medium or to adjust the growth rate of microorganisms according to the rate of addition. The chemostat plays an important role in fermentation engineering, such as the development and production of proteins, vaccines, etc. Hence, the study of the chemostat has attracted the attention of a large number of laboratory technologists, biologists and mathematicians at home and abroad $[2,3,4,5]$, based on its important value in theory and practice. The following deterministic system was studied in [6],

$$
\left\{\begin{array}{l}
S^{\prime}(t)=D\left(S^{0}-S(t)\right)-\frac{1}{\delta} \frac{m S}{a+S} x(t) \\
x^{\prime}(t)=-D x(t)+\frac{m S}{a+S} x(t)
\end{array}\right.
$$

here $S(t), x(t)$ express concentrations of nutrients and microorganisms at time $t$. And all parameters are positive. $S^{0}$ represents the input concentration of nutrient; $D$ is the dilution rate. In the microbial population, this term $\delta$ means conversion rate of nutrient. $a$ is assumed to be the half saturation constant; $m$ stands for the maximum uptake rate of nutrients by microorganisms. [7] has studied the global dynamics of a chemostat model which has multi-competing species and the general functions of describing nutrient uptake. We can obtain the results on multiple competing microbial species in $[8,9,10]$, and results show that the principle of competitive exclusion establishes in the model of competitive chemostat. Based on system (1), we further consider the death of microorganisms in the chemostat, resulting in the following system

$$
\left\{\begin{array}{l}
S^{\prime}(t)=D\left(S^{0}-S(t)\right)-\frac{1}{\delta} \frac{m S}{a+S} x(t) \\
x^{\prime}(t)=\left(\frac{m S}{a+S}-D-D_{1}\right) x(t)
\end{array}\right.
$$

here $D_{1}$ is the death of microorganisms. The system (2) always has a extinction equilibrium point $E^{0}=\left(S^{0}, 0\right)$. And when $D+D_{1} \geq \frac{m S^{0}}{a+S^{0}}, E^{0}$ is global asymptotic stability; when $D+D_{1}<\frac{m S^{0}}{a+S^{0}}$, this system has a positive equilibrium point of global asymptotic stability $E^{*}=\left(S^{*}, x^{*}\right)$, where

$$
\begin{gathered}
S^{*}=\frac{a\left(D+D_{1}\right)}{m-\left(D+D_{1}\right)}, \\
x^{*}=\frac{\delta D}{D+D_{1}}\left(S^{0}-\frac{a\left(D+D_{1}\right)}{m-\left(D+D_{1}\right)}\right) .
\end{gathered}
$$


In addition, biological populations are always affected by periodic fluctuations. However, the previous chemostat models rarely take into account the environmental noises that may be encountered during microbial culture. For example, the laboratory condition control is not strict enough, there are random interference and data measurement errors in the process of information transmission. These stochastic factors will certainly have some influence on the process of microbial culture. In recent, lots of stochastic biological models have been researched and become one of the most important hot spots $[11,12,13,14,15]$. There are two ways to consider the influence of random factors. One is to assume that the influence of random factors on the system is reflected in the influence on some parameters [16]. The other is to assume that the influence of random factors is expressed in the ratio of the deviation from the equilibrium state of the corresponding deterministic system [17]. We will assume that the effects of stochastic factors in the system (2) are shown in the effects on the concentration of nutrients and the mortality of microorganisms, i.e.

we will have

$$
S^{0} \rightarrow S^{0}+\alpha \dot{B}_{1}(t), \quad D_{1} \rightarrow D_{1}+\beta \dot{B}_{2}(t)
$$

$$
\left\{\begin{array}{l}
d S(t)=\left[D\left(S^{0}-S(t)\right)-\frac{1}{\delta} \frac{m S}{a+S} x(t)\right] d t+\alpha D(t) d B_{1}(t), \\
d x(t)=\left[-\left(D+D_{1}\right)+\frac{m S}{a+S}\right] x(t) d t-\beta x(t) d B_{2}(t) .
\end{array}\right.
$$

In the traditional chemostat equations, $S^{0}, D, D_{1}$ can be under the control of the experimenter. People can use the chemostat to industrial engineering and sewage disposal processes, so it is reasonable to change the dilution rate $D$ and the death of microorganisms $D_{1}$ with time. Moreover, because of the change of seasons, etc, other parameters can also exhibit change periodically. Hence we will obtain that

$$
\left\{\begin{array}{l}
d S(t)=\left[D(t)\left(S^{0}-S(t)\right)-\frac{1}{\delta} \frac{m S}{a+S} x(t)\right] d t+\alpha(t) D(t) d B_{1}(t) \\
d x(t)=\left[-\left(D(t)+D_{1}(t)\right)+\frac{m S}{a+S}\right] x(t) d t-\beta(t) x(t) d B_{2}(t) .
\end{array}\right.
$$

We always assume that $\left(\Omega, \digamma,\left\{\digamma_{t}\right\}_{t \geq 0}, \mathbf{P}\right)$ is a complete probability space, and $\left\{\boldsymbol{\digamma}_{t}\right\}$ satisfies the general conditions (i.e. it is right continuous and $\digamma_{0}$ contains all P-null sets) in this paper. Where $B_{i}(t)(i=1,2)$ stand for standard Brownian motions for mutual independence, which define on this space.And $D(t), D_{1}(t), \alpha(t), \beta(t)>0$ are all continuous $\theta$-periodic functions. In this paper, we will prove that the system (3) has unique stationary distribution and ergodic by constructing an appropriate lyapunov function that does not depend on the existence and stability of positive equilibrium. Another purpose of this paper is to obtain the existence of periodic solutions of system (4) according to Khas' minskii[18] theory.

The remaining sections are arranged as follows. In Section 2, we will propose some symbols and auxiliary results. In Section 3, we will prove the system (3) has a unique stationary distribution and achieve appropriate conditions to ensure the existence of a unique $\theta$-periodic solution of system (4). In Section 4, we make numerical simulation to support our results.

\section{Preliminary}

We will often use the following notes. Supposing $f(t)$ is an integrable function $t \in[0, \infty)$, denote $\langle f\rangle_{t}=$ $\frac{1}{t} \int_{0}^{t} f(s) d s$. Supposing function $f(t)$ is a bounded on the interval $[0, \infty)$, express $f^{u}=\sup _{t \in[0, \infty)} f(t)$, $f^{l}=i n f_{t \in[0, \infty)} f(t)$.

Next, we will introduce some preliminaries used in the following sections.

Definition 2.1[18] A stochastic process $X(t)(-\infty<t<+\infty)$ is known as periodic with period $\theta$ if for every finite sequence of the numbers $t_{1}, t_{2}, \cdots, t_{n}$, the joint distribution of random variables $X_{1}, X_{2}, \ldots, X_{n}$ is independent of $\mathrm{h}$, where $h=k \theta(k= \pm 1, \pm 2, \ldots)$.

Remark 2.1[18] Khasminskii has verified that a Markov process $X(t)$ is $\theta$-periodic if and only if its transition probability function is $\theta$-periodic and the function $P_{0}(t, A)=P\{X(t) \in A\}$ satisfies the equation

$$
P_{0}(t, A)=\int_{\mathbb{R}_{l}} P_{0}(s, d x) P(s, x, s+\theta) \equiv P_{0}(s+\theta, A),
$$


for every $A \in \mathfrak{B}$.

Lemma 2.1[18] Suppose there is a bounded domain $U \subset \mathbb{R}^{l}$ with regular boundary $\Gamma$, which satisfies the two conditions:

$(\mathrm{H} 1) F$ is uniformly elliptical in the domain $U$ and some neighborhood thereof, where $F u=b(X) u_{x}+$ $\operatorname{tr}(A(X)) u_{x} x$.

(H2)There has a non-negative $C^{2}$-function $V(X)$ and a positive constant $C$ such that $\mathcal{L} V(X) \leq-C$, for any $X \in \mathbb{R}^{l} \backslash U$.

Then there is a unique stationary distribution $\mu(\cdot)$ of the Markov process $X(t)$, and for any integrable function $f(\cdot)$ with respect to the measure $\mu$, we can obtain that

$$
p\left(\lim _{x \rightarrow \infty} \frac{1}{t} \int_{0}^{t} f(X(t)) d s=\int_{\mathbb{R}_{l}} f(x) \mu(d x)\right)=1
$$

Take the equation into account

$$
X(t)=X\left(t_{0}\right)+\int_{t_{0}}^{t} b(s, X(s)) d s+\sum_{r=1}^{k} \int_{t_{0}}^{t} \sigma_{r}(s, X(s)) d B(s), X \in \mathbb{R}^{l} .
$$

where the vector $b(s, X), \sigma_{1}(s, X), \cdots, \sigma_{k}(s, X)\left(s \in\left(t_{0}, t\right), X \in \mathbb{R}^{l}\right)$ are continuous functions of $(s, X)$ satisfying the conditions:

$$
\begin{array}{r}
|b(s, x)-b(s, y)|+\sum_{r=1}^{k}\left|\sigma_{r}(s, x)-\sigma_{r}(s, y)\right| \leq B|x-y|, \\
|b(s, x)|+\sum_{r=1}^{k}\left|\sigma_{r}(s, x)\right| \leq B(1+|x|),
\end{array}
$$

here $B$ is a constant. Make $U$ be a given open set in $\mathbb{R}^{l}$ and $E=I \times \mathbb{R}^{l}$. Let $C^{2}$ denoted the kind of the functions on $\mathrm{E}$ which are twice continuously differentiable with respect to $x_{1}, \cdots, x_{k}$ and continuously differentiable with respect to $t$.

Lemma 2.2[18] If the coefficients of (5) are $\theta$-periodic in $t$ and satisfy condition (6) in every cylinder $I \times U$. Assume further that there exists a function $V(t, x) \in C^{2}$ in $\mathrm{E}$ which is $\theta$-periodic in $t$, and satisfies the two conditions:

$$
\begin{gathered}
\inf _{|x|>\mathbf{R}} V(t, x) \rightarrow \infty \quad \text { as } \quad \mathbf{R} \rightarrow \infty, \\
\mathcal{L} V \leq-1 \quad \text { outside some compact set, }
\end{gathered}
$$

here we give the operator $\mathcal{L}$

$$
\mathcal{L}=\frac{\partial}{\partial t}+\sum_{i=1}^{l} b_{i}(t, x) \frac{\partial}{\partial x_{i}}+\frac{1}{2} \sum_{i, j=1}^{l} a_{i j}(t, x) \frac{\partial^{2}}{\partial x_{i} y_{j}}, \quad a_{i j}=\sum_{r=1}^{l} \sigma_{r}^{i}(t, x) \sigma_{r}^{j}(t, x) .
$$

Then (5) has a solution of $\theta$-periodic Markov process.

\section{Main Results}

In this section, firstly, We will prove the system (4) has a unique global solution by using the comparison theorem. Then, we will prove the system (3) has a unique stationary distribution by constructing a Lyapunov function. Finally, we will find the sufficient conditions to prove that system (4) has a nontrivial positive periodic solutions. 
Theorem 3.1 There exists a unique global positive solution $(S(t), x(t)) \in \mathbb{R}_{+}^{2}$ of system (4) on $t \geq 0$ for any initial value $(S(0), x(0)) \in \mathbb{R}_{+}^{2}$.

Proof. Because the coefficients of system (4) meet the local Lipschitz conditions, system (4) has a unique local solution $(S(t), x(t))$ on $t \in\left[0, \tau_{e}\right)$, here $t \in\left[0, \tau_{e}\right)$ is the explosion time, for any initial value. We just should testify $\tau_{e}=\infty$ to prove that this solution is global. Because of $(S(t), x(t)) \in \mathbb{R}_{+}^{2}$, we have

$$
d S(t) \leq D^{u}\left(S^{0}-S(t)\right) d t+\alpha^{u} D^{u} d B_{1}(t)
$$

and

$$
d x(t) \leq\left(m-D_{1}^{l}-D^{l}\right) x(t) d t-\beta^{l} x(t) d B_{1}(t)
$$

We let $\Psi(t)$ and $\Phi(t)$ be solutions of the following equations, respectively.

$$
\begin{gathered}
\left\{\begin{array}{l}
\Psi(t)=D^{u}\left(S^{0}-\Psi(t)\right) d t+\alpha^{u} D^{u} d B_{1}(t), \\
\Psi(0)=S(0)=S^{0} .
\end{array}\right. \\
\left\{\begin{array}{l}
\Phi(t)=\left(m-D_{1}^{l}-D^{l}\right) \Phi(t) d t-\beta^{l} x(t) d B_{1}(t), \\
\Phi(0)=x(0) .
\end{array}\right.
\end{gathered}
$$

According to the comparison theorem, we have $S(t) \leq \Psi(t), x(t) \leq \Phi(t), t \in\left[0, \tau_{e}\right) \quad$ a.s. Analogously, we can obtain that

$$
d S(t) \geq-D^{l} S(t) d t+\alpha^{l} D^{l} d B_{1}(t)
$$

and

$$
d x(t) \geq-\left(D_{1}^{u}+D^{u}\right) x(t) d t-\beta^{u} x(t) d B_{1}(t)
$$

We let $\varphi(t)$ and $\phi(t)$ be solutions of the following equations, respectively.

$$
\begin{gathered}
\left\{\begin{array}{l}
\varphi(t)=-D^{l} S(t) d t+\alpha^{l} D^{l} d B_{1}(t), \\
\varphi(0)=S(0)=S^{0} .
\end{array}\right. \\
\left\{\begin{array}{l}
\phi(t)=-\left(D_{1}^{u}+D^{u}\right) x(t) d t-\beta^{u} x(t) d B_{1}(t), \\
\Phi(0)=x(0) .
\end{array}\right.
\end{gathered}
$$

In the same way, we have $S(t) \geq \varphi(t), x(t) \geq \phi(t), t \in\left[0, \tau_{e}\right)$ a.s. From what has been discussed above, we have

$$
\varphi(t) \leq S(t) \leq \Psi(t), \quad \phi(t) \leq x(t) \leq \Phi(t), \quad t \in\left[0, \tau_{e}\right) \quad \text { a.s. }
$$

Furthermore,there has unique global positive solutions of the equations $[9,10,11,12]$. In other words, for any $t \in[0, \infty), \Psi(t), \Phi(t), \varphi(t), \phi(t)$ are true, so $\tau_{e}=\infty$. That is the proof.

Theorem 3.2 Suppose $D S^{0}>\frac{(\alpha D)^{2}}{2}, D+D_{1}>\frac{m S^{0}}{a+S^{0}}, D+D_{1}+2 \beta^{2}>m$. Then for any initial value, the system $(3)$ has a unique stationary distribution $\mu(\cdot)$, and it has ergodic property.

Proof. For any initial value $(S(0), x(0)) \in \mathbb{R}_{+}^{2}$, there has a unique solution of system (3). From Lemma 2.1 , in order to prove Theorem 3.2, we should find a $C^{2}$-function $V(t, x)$ and a bounded domain $U \in \mathbb{R}_{+}^{2}$ that make conditions (H1) and (H2) true. Firstly, we construct a Lyapunov function $V: \mathbb{R}_{+}^{2} \rightarrow \mathbb{R}_{+}$

$$
V(S, x)=-S^{0} \log \frac{S}{S^{0}}+S+f(x)-S^{0}-f(\sqrt{\delta})
$$

where $f(x)=\frac{1}{\delta} x-\frac{2}{\sqrt{\delta}} \log x-\frac{1}{x}$. 
We can get the following inequality by the Itô's formula,

$$
\begin{aligned}
\mathcal{L} V & =D\left(S^{0}-S\right)-\frac{1}{\delta} \frac{m S}{a+S} x-\frac{S^{0}}{S} D\left(S^{0}-S\right)+\frac{S^{0}}{S} \frac{1}{\delta} \frac{m S}{a+S} x+\frac{S^{0}(\alpha D)^{2}}{2 S^{2}} \\
& +\frac{1}{\delta} \frac{m S}{a+S} x-\frac{1}{\delta}\left(D+D_{1}\right) x-\frac{2}{\sqrt{\delta}}\left(\frac{m S}{a+S}-D-D_{1}\right)+\frac{1}{x}\left(\frac{m S}{a+S}-D-D_{1}-2 \beta^{2}\right)+\frac{2}{\sqrt{\delta}} \beta^{2} \\
& \leq-D S-\frac{S^{0}}{S}\left(D S^{0}-\frac{(\alpha D)^{2}}{2}\right)+\frac{S^{0}}{\delta} \frac{m}{a+S} x+2 D S^{0}-\frac{1}{\delta}\left(D+D_{1}\right) x-\frac{2}{\sqrt{\delta}}\left(\frac{m S}{a+S}-D-D_{1}\right) \\
& +\frac{1}{x}\left(m-D-D_{1}-2 \beta^{2}\right)+\frac{2}{\sqrt{\delta}} \beta^{2}
\end{aligned}
$$

Here we define $p_{1}(S)=\frac{m S}{a+S}$ and $p_{2}(S)=\frac{m}{a+S}$. Since $p_{i}(S)(i=1,2)$ is continuously differentiable and $p_{i}(0)=0, \quad p_{i}(S)>0(i=1,2) \quad$ for $\quad S>0$, we have

$$
p_{1}^{\prime}(S)=\frac{m a}{(a+S)^{2}}>0, \quad p_{2}^{\prime}(S)=-\frac{m}{(a+S)^{2}}<0 \quad \text { for } S>0 .
$$

Then,

Therefore,

$$
p_{1}(S)>p_{1}\left(S^{0}\right)=\frac{m S^{0}}{a+S^{0}}, \quad p_{2}(S)<p_{2}\left(S^{0}\right)=\frac{m}{a+S^{o}}:=c^{0} .
$$

$$
\begin{aligned}
\mathcal{L} V & \leq-D S-\frac{S^{0}}{S}\left(D S^{0}-\frac{(\alpha D)^{2}}{2}\right)+\frac{S^{0}}{\delta} \frac{m}{a+S^{0}} x+2 D S^{0}-\frac{1}{\delta}\left(D+D_{1}\right) x \\
& -\frac{2}{\sqrt{\delta}}\left(\frac{m S^{0}}{a+S^{0}}-D-D_{1}\right)+\frac{1}{x}\left(m-D-D_{1}-2 \beta^{2}\right)+\frac{2}{\sqrt{\delta}} \beta^{2} \\
& =\lambda-D S-\frac{S^{0}}{S}\left(D S^{0}-\frac{(\alpha D)^{2}}{2}\right)-\frac{1}{\delta}\left(D+D_{1}-\frac{m S^{0}}{a+S^{0}}\right) x-\frac{1}{x}\left(D+D_{1}+2 \beta^{2}-m\right) \\
& +2 D S^{0}-\frac{2}{\sqrt{\delta}}\left(\frac{m S^{0}}{a+S^{0}}-D-D_{1}\right) .
\end{aligned}
$$

here $\lambda=2 D S^{0}-\frac{2}{\sqrt{\delta}}\left(\frac{m S^{0}}{a+S^{0}}-D-D_{1}\right)+\frac{2}{\sqrt{\delta}} \beta^{2}$

Then we choose sufficiently small $\varepsilon_{1}, \varepsilon_{2}$ such that

$$
\begin{gathered}
0<\varepsilon_{1}<\min \left\{\frac{S^{0}}{\lambda+1}\left(D S^{0}-\frac{(\alpha D)^{2}}{2}\right), \frac{D}{\lambda+1}\right\} \\
0<\varepsilon_{2}<\min \left\{\frac{D+D_{1}+2 \beta^{2}-m}{\lambda+1}, \frac{D+D_{1}-\frac{m S^{0}}{a+S^{0}}}{\lambda+1}\right\} .
\end{gathered}
$$

Next we discuss the bounded open subset

$$
D_{\varepsilon_{1}, 2}=\left\{(S, x) \mid \varepsilon_{1}<S<\frac{1}{\varepsilon_{1}}, \varepsilon_{2}<x<\frac{1}{\varepsilon_{2}}\right\} .
$$

Define

$$
\begin{array}{ll}
D_{\varepsilon_{1,2}}^{1}=\left\{(S, x) \in \mathbb{R}_{+}^{2} \mid 0<S<\varepsilon_{1}\right\} & D_{\varepsilon_{1,2}}^{2}=\left\{(S, x) \in \mathbb{R}_{+}^{2} \mid S>\frac{1}{\varepsilon_{1}}\right\}, \\
D_{\varepsilon_{1,2}}^{3}=\left\{(S, x) \in \mathbb{R}_{+}^{2} \mid 0<x<\varepsilon_{2}\right\} & D_{\varepsilon_{1,2}}^{4}=\left\{(S, x) \in \mathbb{R}_{+}^{2} \mid x>\frac{1}{\varepsilon_{2}}\right\} .
\end{array}
$$

Easily, $D_{\varepsilon_{1,2}}^{c}=D_{\varepsilon_{1}, 2}^{1} \cup D_{\varepsilon_{1,2}}^{2} \cup D_{\varepsilon_{1,2}}^{3} \cup D_{\varepsilon_{1,2}}^{4}$. When $(S, x) \in D_{\varepsilon_{1,2}}^{1}$, we can obtain that

$$
\mathcal{L} V \leq \lambda-\frac{S^{0}}{s}\left(D S^{0}-\frac{(\alpha D)^{2}}{2}\right) \leq \lambda-\frac{S^{0}}{\varepsilon}\left(D S^{0}-\frac{(\alpha D)^{2}}{2}\right)<-1 .
$$


In a similar way, we can find that $\mathcal{L} V \leq-1$ in $D_{\varepsilon_{1,2}}^{2}, D_{\varepsilon_{1,2}}^{3}, D_{\varepsilon_{1,2}}^{4}$, respectively. Hence, for any $(S, x) \in$ $D_{\varepsilon_{1,2}}^{c}$, we have $\mathcal{L} V \leq-1$. Then we let $U$ to be a neighborhood of $D_{\varepsilon_{1,2}}^{c}$ with $\bar{U} \subset \mathbb{R}_{+}^{2}$. It is easy to get $\mathcal{L} V \leq-1$, for $(S, x) \in \mathbb{R}_{+}^{2} \backslash U$. Therefore, the condition (H2) of Lemma 2.1. is true. What's more, we take $M=\min _{(S, x) \in \bar{U}}\left\{\alpha^{2} D^{2}, \beta^{2} x^{2}\right\}$ such that

$$
\sum_{i, j=1,2}^{2} a_{i} j(S, x) \xi_{i} \xi_{j}=\alpha^{2} D^{2} \xi_{1}^{2}+\beta^{2} x^{2} \xi_{2}^{2} \geq M\|\xi\|^{2}, \quad f o r(S, x) \in \bar{U}, \quad \xi=\left(\xi_{1}, \xi_{2}\right) \in \mathbb{R}_{+}^{2} .
$$

In other words, the condition (H1) of Lemma 2.1 is true. Hence, we can see that the system (3) has a unique stationary distribution and ergodic property by Lemma 2.1. That's the proof.

Theorem 3.3 If $\left\langle R_{0}\right\rangle_{\theta}>0$, system (4) has a nontrivial positive $\theta$-periodic solution. Where $R(t):=$ $\frac{m S^{0}}{a+S^{0}}-2 D(t)-D_{1}(t)-\frac{1}{2} \beta^{2}(t)$

Proof. For arbitrary initial value $(S(0), x(0)) \in \mathbb{R}_{+}^{2}$, according to Theorem 3.1 , system (4) exists a unique global positive solution. Hence we let $\mathbb{R}_{+}^{2}$ as the whole space. Obviously, the coefficients of the system (4) meet (6). As we know from Lemma 2.2, if we want to prove Theorem 3.3, all we have to do is just seek out a $C^{2}$-function $V(t, x)$ and a closed set $U \in \mathbb{R}_{+}^{2}$ that make conditions $(7)$ and (8) true. First of all, we choose a constant $c=\max \left\{\left(\alpha^{u}\right)^{2},\left(D_{1}^{u}\right)^{2}+\left(\beta^{u}\right)^{2}\right\}$ such that

(A)

$$
D^{l}-\frac{1}{2}-c>0
$$

Next, a positive constant $M$ big enough is be taken to make that

(B)

$$
f^{u}-M\langle R\rangle_{\theta} \leq-2 .
$$

$f(S, x)$ is a function described later in $(\boldsymbol{C})$.

Denote $V_{1}=-\log \frac{S}{S^{0}}-\log x-x, V_{2}=\left(S-S^{0}+\frac{1}{\delta} x\right)^{2}$. Therefore we get a $C^{2}$-function:

$$
V=M\left(V_{1}+\omega(t)\right)+V_{2},
$$

where function $\omega(t), \quad t \in[0,+\infty)$ satisfies

$$
\dot{\omega}(t)=R(t)-\langle R\rangle_{\theta}, \quad \omega(0)=0,
$$

It is obvious to see that $\omega(t)$ is a $\theta$-periodic function $t \in[0,+\infty)$ and $V(t, S, x)$ is a $\theta$-periodic in $t$ which satisfies condition (7). Then we should prove the condition (8).

By the Itô's formula, we have

$$
\begin{aligned}
\mathcal{L} V_{1} & =-\frac{1}{S}\left[D(t)\left(S^{0}-S\right)-\frac{1}{\delta} \frac{m S}{a+S} x\right]+\frac{1}{2 S^{2}} \alpha^{2}(t) D^{2}(t) \\
& -\frac{m S}{a+S}+D(t)+D_{1}(t)+\frac{1}{2} \beta^{2}(t)+\left(\frac{m S}{a+S}-D(t)-D_{1}(t)\right) x \\
& =-\frac{D(t) S^{0}}{S}+2 D(t)+D_{1}(t)+\frac{1}{\delta} \frac{m}{a+S} x+\frac{1}{2 S^{2}} \alpha^{2}(t) D^{2}(t) \\
& -\frac{m S}{a+S}+\frac{1}{2} \beta^{2}(t)+\left(\frac{m S}{a+S}-D(t)-D_{1}(t)\right) x \\
& \leq-\frac{D^{l} S^{0}}{S}+\frac{\left(\alpha^{u} D^{u}\right)^{2}}{2 S}-\frac{m S}{a+S}+\frac{1}{\delta} \frac{m}{a+S} x+\left(m-D^{l}-D_{1}^{l}\right) x+2 D(t)+D_{1}(t)+\frac{1}{2} \beta^{2}(t) \\
& \leq-\frac{D^{l} S^{0}-\left(\alpha^{u} D^{u}\right)^{2}}{2 S}+\frac{c^{0}}{\delta} x+\left(m-D^{l}-D_{1}^{l}\right) x-\left[\frac{m S^{0}}{a+S^{0}}-2 D(t)-D_{1}(t)-\frac{1}{2} \beta^{2}(t)\right] .
\end{aligned}
$$

Next,

$$
\mathcal{L}\left(V_{1}+\omega(t)\right) \leq-\langle R\rangle_{\theta}-\frac{D^{l} S^{0}-\left(\alpha^{u} D^{u}\right)^{2}}{2 S}+\frac{c^{0}}{\delta} x+\left(m-D^{l}-D_{1}^{l}\right) x
$$


Let us consider the $V_{2}$, using the Itô's formula again, we have

$$
\begin{aligned}
\mathcal{L} V_{2} & =2\left(S-S^{0}+\frac{1}{\delta} x\right)\left[D(t)\left(S-S^{0}\right)-\frac{1}{\delta} \frac{m S}{a+S} x+\frac{1}{\delta} x\left(\frac{m S}{a+S}-D(t)-D_{1}(t)\right)\right]+\alpha^{2}(t) D^{2}(t)+\frac{1}{\delta^{2}} \beta^{2}(t) x^{2}, \\
& =-2\left(S-S^{0}\right)^{2} D(t)-2\left(S-S^{0}\right) \frac{1}{\delta} x\left(D(t)+D_{1}(t)\right)-\frac{2}{\delta} x D(t)\left(S-S^{0}\right) \\
& -2\left(\frac{1}{\delta} x\right)^{2}\left(D(t)+D_{1}(t)+\alpha^{2}(t) D^{2}(t)+\frac{1}{\delta^{2}} \beta^{2}(t) x^{2}\right) \\
& =-2 D(t)\left[\left(S-S^{0}\right)^{2}+\frac{2}{\delta} x\left(S-S^{0}\right)+\left(\frac{1}{\delta} x\right)^{2}\right]-2\left(S-S^{0}\right) \frac{1}{\delta} x D_{1}(t) \\
& -2\left(\frac{1}{\delta} x\right)^{2} D_{1}(t)+\alpha^{2}(t) D^{2}(t)+\frac{1}{\delta^{2}} \beta^{2}(t) x^{2}, \\
& =-2 D(t)\left(S-S^{0}+\frac{x}{\delta}\right)^{2}-2\left(S-S^{0}+\frac{x}{\delta}\right) D_{1}(t) \frac{x}{\delta}+\alpha^{2}(t) D^{2}(t)+\frac{1}{\delta^{2}} \beta^{2}(t) x^{2} .
\end{aligned}
$$

Using the fact that $a^{2}+b^{2} \geq-2 a b, \quad$ for any $a, b \in \mathbb{R}$, we can see

$$
\begin{aligned}
\mathcal{L} V_{2} & \leq-2 D(t)\left(S-S^{0}+\frac{x}{\delta}\right)^{2}+\left(S-S^{0}+\frac{x}{\delta}\right)^{2}+\left(D_{1}(t) \frac{x}{\delta}\right)^{2}+\alpha^{2}(t) D^{2}(t)+\frac{1}{\delta^{2}} \beta^{2}(t) x^{2}, \\
& =-2\left(D(t)-\frac{1}{2}\right)\left(S-S^{0}+\frac{x}{\delta}\right)^{2}+\alpha^{2}(t) D^{2}(t)+\frac{D_{1}^{2}(t)+\beta^{2}(t)}{\delta^{2}} x^{2}, \\
& \leq-2\left(D(t)-\frac{1}{2}\right)\left(S-S^{0}+\frac{x}{\delta}\right)^{2}+\alpha^{2}(t) D^{2}(t)+\frac{\left(D_{1}^{u}\right)^{2}+\left(\beta^{u}\right)^{2}}{\delta^{2}} x^{2} .
\end{aligned}
$$

Pay attention to the

$$
\begin{aligned}
\frac{\left(D_{1}^{u}\right)^{2}+\left(\beta^{u}\right)^{2}}{\delta^{2}} x^{2} & \leq\left(\alpha^{u}\right)^{2} S^{2}+\frac{\left(D_{1}^{u}\right)^{2}+\left(\beta^{u}\right)^{2}}{\delta^{2}} x^{2} \\
& \leq\left(\alpha^{u}\right)^{2} S^{2} \frac{\left(D_{1}^{u}\right)^{2}+\left(\beta^{u}\right)^{2}}{\delta^{2}} x^{2} \\
& \leq \max \left\{\left(\alpha^{u}\right)^{2},\left(D_{1}^{u}\right)^{2}+\left(\beta^{u}\right)^{2}\right\}\left(S+\frac{x}{\delta}\right)^{2} \\
& =c\left(S+\frac{x}{\delta}\right)^{2} .
\end{aligned}
$$

Hence

$$
\begin{aligned}
\mathcal{L} V_{2} & \leq-2\left(D(t)-\frac{1}{2}\right)\left(S-S^{0}+\frac{x}{\delta}\right)^{2}+\alpha^{2}(t) D^{2}(t)+c\left(S+\frac{x}{\delta}\right)^{2} \\
& \leq-2\left(D(t)-\frac{1}{2}\right)\left(S-S^{0}+\frac{x}{\delta}\right)^{2}+\alpha^{2}(t) D^{2}(t)+c\left[2\left(S-S^{0}+\frac{x}{\delta}\right)^{2}+2\left(S^{0}\right)^{2}\right] \\
& =-2\left(D(t)-\frac{1}{2}\right)\left(S-S^{0}+\frac{x}{\delta}\right)^{2}+\alpha^{2}(t) D^{2}(t)+2 c\left(S-S^{0}+\frac{x}{\delta}\right)^{2}+2 c\left(S^{0}\right)^{2} \\
& =-2\left(D(t)-\frac{1}{2}-c\right)\left(S-S^{0}+\frac{x}{\delta}\right)^{2}+\alpha^{2}(t) D^{2}(t)+2 c\left(S^{0}\right)^{2} \\
& \leq-2\left(D^{l}-\frac{1}{2}-c\right)\left(S-S^{0}+\frac{x}{\delta}\right)^{2}+\left(\alpha^{u}\right)^{2}\left(D^{u}\right)^{2}+2 c\left(S^{0}\right)^{2} .
\end{aligned}
$$

This combined with the (15)and (16), showing that

$$
\begin{aligned}
\mathcal{L} V & \leq M\left[-\langle R\rangle_{\theta}-\frac{D^{l} S^{0}-\left(\alpha^{u} D^{u}\right)^{2}}{2 S}+\frac{c^{0}}{\delta} x+\left(m-D^{l}-D_{1}^{l}\right) x\right] \\
& -2\left(D^{l}-\frac{1}{2}-c\right)\left(S-S^{0}+\frac{x}{\delta}\right)^{2}+\left(\alpha^{u}\right)^{2}\left(D^{u}\right)^{2}, \\
& =f(S, x)+g(x) .
\end{aligned}
$$


here $(\boldsymbol{C})$

$$
\left\{\begin{array}{l}
f(S, x)=-2\left(D^{l}-\frac{1}{2}-c\right)\left(S-S^{0}+\frac{x}{\delta}\right)^{2}-M \frac{D^{l} S^{0}-\left(\alpha^{u} D^{u}\right)^{2}}{2 S}+\left(\alpha^{u}\right)^{2}\left(D^{u}\right)^{2}+2 c\left(S^{0}\right)^{2}, \\
g(x)=-M\langle R\rangle_{\theta}+M\left(m-D^{l}-D_{1}^{l}+\frac{c^{0}}{\delta}\right) x .
\end{array}\right.
$$

We can see that

$$
\begin{gathered}
f(+\infty, x)+g^{u} \rightarrow-\infty, \quad \text { as } \quad S \rightarrow+\infty, \\
f(S,+\infty)+g(+\infty) \rightarrow-\infty, \quad \text { as } \quad x \rightarrow+\infty,
\end{gathered}
$$

When $D^{l} S^{0}-\left(\alpha^{u} D^{u}\right)^{2}>0$

$$
f\left(0^{+}, x\right)+g^{u} \rightarrow-\infty, \quad \text { as } \quad S \rightarrow 0^{+} .
$$

All of these cases make for $\mathcal{L} V<-1$. From $\boldsymbol{B}$

$$
f\left(S, 0^{+}\right)+g\left(0^{+}\right) \rightarrow f^{u}-M\langle R\rangle_{\theta}, \quad \text { as } \quad x \rightarrow 0^{+} .
$$

Then we will choose $\varepsilon$ small enough to make $U=\left[\varepsilon, \frac{1}{\varepsilon}\right] \times\left[\varepsilon, \frac{1}{\varepsilon}\right]$. Thus it can be seen that

$$
\mathcal{L} V<-1, \quad(S, x) \in \mathbb{R}_{+}^{2} \backslash U .
$$

That's the proof.

In proving theorem 3.2 and 3.3, we found that the most difficult problem was to construct Lyapunov function that satisfies Lemma 2.1 and 2.2 respectively.

\section{Numerical Simulations}

We will use numerical methods to simulate the results in this section. As shown in the following figures, the blue lines stand for the solution of stochastic system; the red lines are the solution of the relevant deterministic system.

Firstly, we choose parameters $S^{0}=1, m=2, a=4, \delta=0.8, D=1.3, D_{1}=0.8, \alpha=0.016, \beta=0.4$, according to Theorem 3.2, there has a stationary distribution of the system (3). The numerical simulations in Fig. 1.
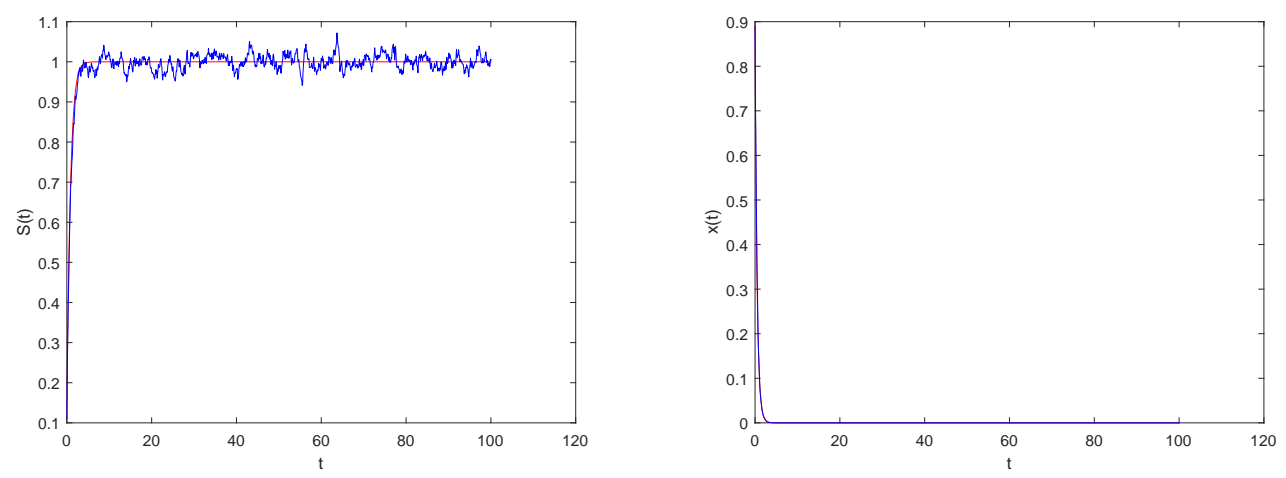

Figure 1. The solution of system (3) and its relevant undistributed system with initial conditions $(0)=$ $0.11, x(0)=0.89$.

Next, we will denote $D(t)=1.8+0.2 \sin t, D_{1}(t)=0.6+0.2 \cos t, S^{0}=1, m=3, a=0.2, \delta=0.8, m_{0}=$ $0.5, M=50, c_{2}=0.7, \alpha(t)=0.2+0.05 \cos t, \beta(t)=0.2+0.05 \cos t$. 
Then $\langle R\rangle_{2 \pi}>0$. Thus system (4) has a $2 \pi$-periodic solution. The numerical simulations in Fig. 2,3,4 sustain these result distinctly. And in Fig. 4, the noise intensities become smaller, here we choose $\alpha(t)=0.01+0.005 \cos t, \beta(t)=0.01+0.005 \cos t$.
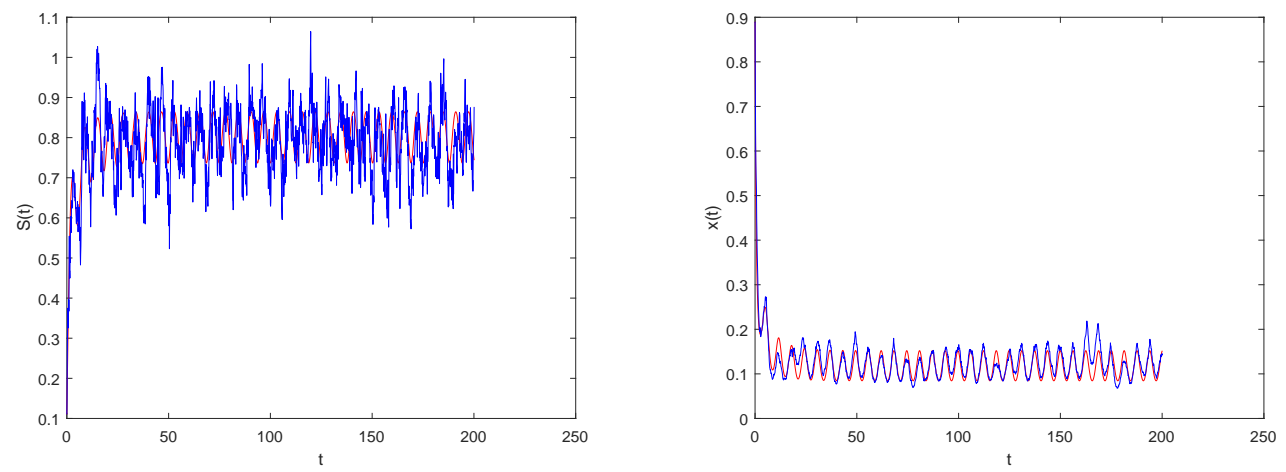

Figure 2. The periodic solution of system (4) and its relevant undistributed system with initial conditions $(0)=0.11, x(0)=0.89$.
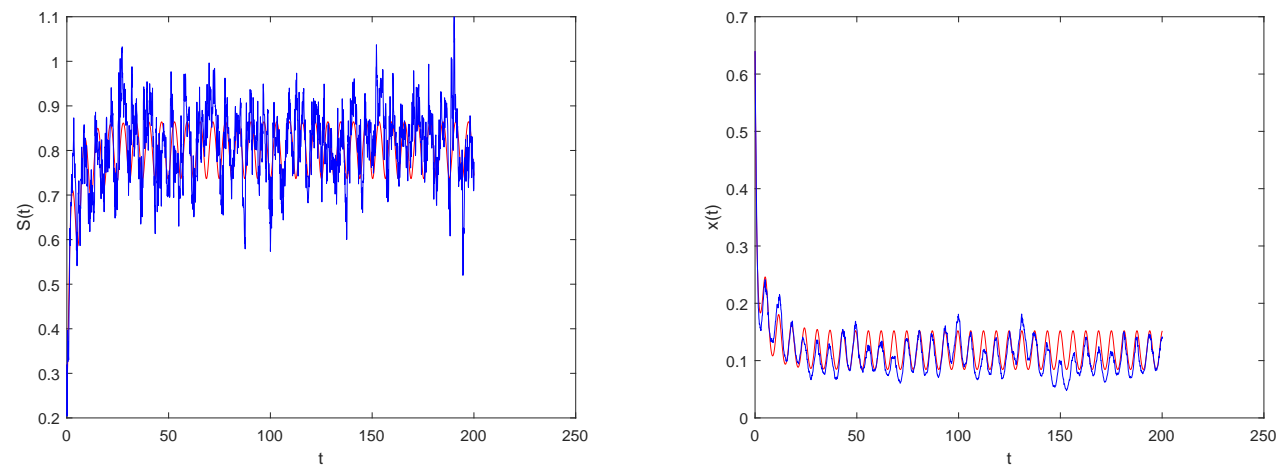

Figure 3. The periodic solution of system (4) and its relevant undistributed system with initial values $S(0)=$ $0.32, x(0)=0.64$. 

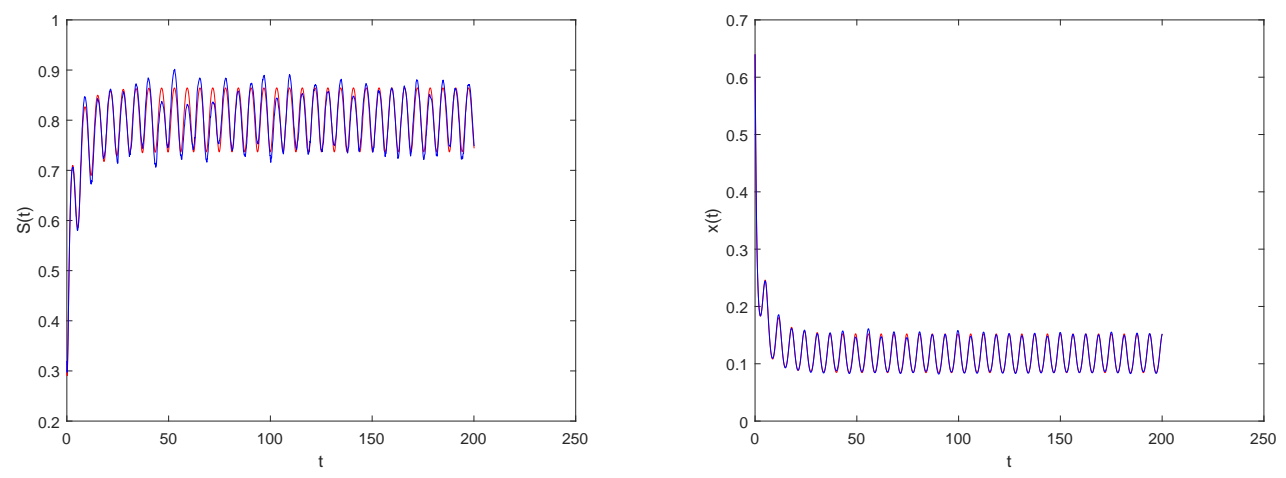

Figure 4. The periodic solution of system (4) and its relevant undistributed system with initial values $S(0)=$ $0.32, x(0)=0.64$.

As can be seen from figure 2 and figure 3, for arbitrary initial value, the solution of deterministic system will go into the periodic orbit after a cycle of time. According to Fig. 3 and 4, the noise intensity is small enough so that the stochastic system's solution will fluctuate near the periodic orbit.

\section{Conclusion}

In this paper, we discuss a kind of stochastic chemostat model with Monod's functional response, in which concentration of nutrients and mortality of microorganisms are affected by white noise. We have proven that system (3) has a unique stationary distribution and it is ergodic, when $D S^{0}>\frac{(\alpha D)^{2}}{2}, D+D_{1}>$ $\frac{m S^{0}}{a+S^{0}}, D+D_{1}+2 \beta^{2}>m$ (see in Theorem 3.2.). The presence of a stationary distribution implies some degree of stochastic stability. By Theorem 3.3., we have obtained that if $\left.\langle R\rangle_{\theta}\right\rangle 0$, system (4) exists a unique nontrivial positive $\theta$-periodic solution. When the density of white noise is small enough, the solutions of stochastic systems are less perturbed. Therefore, if we control the white noise reasonably, we can make the chemostat achieve the ideal effect.

\section{References}

1. Smith H L, Waltman P. The Theory of Chemostat: Dynamics of Microbial Competition J. Am. Chem. Soc. $1995,117,11616[\mathrm{~J}]$.

2. Zhang T, Zhang T, Meng X. Stability analysis of a chemostat model with maintenance energy[J]. Applied Mathematics Letters, 2017, 68: 1-7.

3. Butler G J, Hsu S B, Waltman P. A mathematical model of the chemostat with periodic washout rate[J]. SIAM Journal on Applied Mathematics, 1985, 45(3): 435-449.

4. Weedermann M, Seo G, Wolkowicz G. Mathematical model of anaerobic digestion in a chemostat: effects of syntrophy and inhibition[J]. Journal of biological dynamics, 2013, 7(1): 59-85.

5. Stephanopoulos G, Lapudis G R. Chemostat dynamics of plasmid-bearing, plasmid-free mixed recombinant cultures[J]. Chemical Engineering Science, 1988, 43(1): 49-57.

6. Ji C, Jiang D, Shi N. A note on a predatoríCprey model with modified LeslieĺCGower and Holling-type II schemes with stochastic perturbation[J]. Journal of Mathematical Analysis and Applications, 2011, 377(1): 435-440.

7. Butler G J, Wolkowicz G S K. A mathematical model of the chemostat with a general class of functions describing nutrient uptake[J]. SIAM Journal on applied mathematics, 1985, 45(1): 138-151.

8. Li B. Global asymptotic behavior of the chemostat: general response functions and different removal rates[J]. SIAM Journal on Applied Mathematics, 1998, 59(2): 411-422.

9. Liu S, Wang X, Wang L, et al. Competitive exclusion in delayed chemostat models with differential removal rates[J]. SIAM Journal on Applied Mathematics, 2014, 74(3): 634-648.

10. Wolkowicz G S K, Lu Z. Global dynamics of a mathematical model of competition in the chemostat: general response functions and differential death rates[J]. SIAM Journal on Applied Mathematics, 1992, 52(1): 222233. 
11. Zhang T, Chen Z, Han M. Dynamical analysis of a stochastic model for cascaded continuous flow bioreactors[J]. Journal of Mathematical Chemistry, 2014, 52(5): 1441-1459.

12. Zhang X, Jiang D, Hayat T, et al. Dynamics of a stochastic SIS model with double epidemic diseases driven by Llęvy jumps[J]. Physica A: Statistical Mechanics and its Applications, 2017, 471: 767-777.

13. Meng X, Zhao S, Feng T, et al. Dynamics of a novel nonlinear stochastic SIS epidemic model with double epidemic hypothesis[J]. Journal of Mathematical Analysis and Applications, 2016, 433(1): 227-242.

14. Zhao Y, Jiang D, OąŕRegan D. The extinction and persistence of the stochastic SIS epidemic model with vaccination[J]. Physica A: Statistical Mechanics and Its Applications, 2013, 392(20): 4916-4927.

15. Ji C, Jiang D, Shi N. Analysis of a predatoríCprey model with modified LeslieĺCGower and Holling-type II schemes with stochastic perturbation[J]. Journal of Mathematical Analysis and Applications, 2009, 359(2): 482-498.

16. Jiang $\mathrm{D}, \mathrm{Yu} \mathrm{J}, \mathrm{Ji} \mathrm{C}$, et al. Asymptotic behavior of global positive solution to a stochastic SIR model[J]. Mathematical and Computer Modelling, 2011, 54(1-2): 221-232.

17. Ji C, Jiang D. Dynamics of a stochastic density dependent predatoríCprey system with BeddingtoníCDeAngelis functional response[J]. Journal of Mathematical Analysis and Applications, 2011, 381(1): 441-453.

18. Khasminskii R. Stochastic stability of differential equations[M]. Springer Science Business Media, 2011. 